\title{
GENETICS OF OBESITY IN ZUCKER RATS AND KOLETSKY RATS
}

\author{
TERENCE T. YEN, WALTER N. SHAW and PAO-LO YU* \\ The Lilly Research Laboratories, Eli Lilly and Company, Indianapolis, Indiana 46206 and \\ * Department of Medical Genetics, Indiana University School of Medicine, Indianapolis, Indiana 46202
}

Received 29.xi.76

\section{SUMMARY}

The breeding data on Zucker rats and on Koletsky rats confirm that the obesity in these two strains of rats is inherited recessively and results from single gene mutations. Mating a Zucker heterozygote to a Koletsky heterozygote produced obese $F_{1}$ progeny. Inter-stock breeding results indicate that the obesity in the Zucker-Koletsky hybrid stock is also inherited in a recessive manner. The gene that controls obesity in the Zucker rats, fatty $(\mathrm{fa})$, and the gene that controls obesity in the Koletsky rats, $f$, are thus alleles at the same locus. We propose that $f$ be renamed $f a^{k}$ until it can be proven that $f a$ and $f a^{k}$ are identical.

\section{IntroduGtion}

To date, only two spontaneous mutations have been discovered in rats that cause obesity. The first one was described by Zucker and Zucker (1961) who named the gene fatty $(f a)$. The second one was more recently described by Koletsky (1973) who proposed to use the letter $F$ for the dominant allele and the letter $f$ for the recessive allele.

The mutation fatty first appeared in a cross between Sherman and Merck stock $M$ rats (Zucker and Zucker, 1963). The affected rats were obese, hyperlipemic and hyperinsulinemic (Bray and York, 1971). The Koletsky obese rats arose from matings between Okamoto-Aoki spontaneously hypertensive rats of Wistar/Kyoto strain (Okamoto and Aoki, 1963) and Sprague-Dawley rats. The affected rats were hypertensive and obese. They also had marked hypertriglyceridemia, moderate hypercholesterolemia and a lipoprotein pattern resembling that of human type IV hyperlipoproteinemia (Koletsky, 1975). All Koletsky obese rats had proteinuria as a result of increased permeability of glomerular lesions (Koletsky, 1975). About half of the Koletsky obese rats developed vascular lesions similar to those of human atherosclerosis (Koletsky, 1975).

Based on the initial breeding data, Zucker and Zucker (1961) proposed that the obesity of the fatty rats resulted from a single gene mutation, which was inherited in a recessive fashion. Koletsky (1973) suggested that his obese rats also resulted from a single gene mutation which was also inherited in a recessive manner; however, no breeding data have been published on Koletsky rats.

We wish to report in this communication that our breeding data on both Zucker rats and Koletsky rats support both Zucker and Zucker and

Reprint requests to: Dr T. T. Yen, Biological Research Division, Lilly Research Laboratories, Eli Lilly and Company, 307 East McCarty Street, Indianapolis, Indiana 46206. 
Koletsky's single gene, recessive inheritance hypotheses. In addition, our data indicate that the two mutations occurred at the same locus.

Some of the information contained herewith has been reported in a preliminary communication (Yen, Shaw and Yu, 1976).

\section{Materials and methods}

Our original Zucker breeding stock was obtained from Dr L. M. Zucker in 1969. Our original Koletsky breeding stock was obtained from Dr S. Koletsky in 1974. Each stock was maintained by outbreeding within the closed colony. Brother $\times$ sister matings and back-crosses were avoided. Each mating always consisted of only one male and one female rat. The male rat was removed from the breeding cage before the litter was born. Since the inception of both breeding programmes, no new rats were introduced into either colony from outside. All rats in both colonies were descendants from our original breeding stocks.

Rats were kept in plastic cages with bedding. Water and food were available ad libitum. The Zucker rats were fed Camm Research Rat and Mouse Ration (Camm Research Institute, Wayne, New Jersey). The Koletsky rats were fed Purina Laboratory Chow (Ralston Purina Co., St Louis, Missouri). The ambient temperature in each animal room was maintained at about $25^{\circ} \mathrm{C}$. The photoperiod was controlled to provide dark from 1800 to 0600 hours and light from 0600 to 1800 hours.

Breeding results were analysed by Morton's method of segregation analysis (Morton, 1958 a and 1958b) and a program "SEGRAN" developed for the computer.

The term "carrier" in this communication refers to a rat determined to be a heterozygote by having produced at least one obese rat in previous litters. The term " unknown " refers to a rat which had not been identified as being a heterozygote before.

\section{Results}

All the breeding data are shown in table 1.

For both Zucker rats and Koletsky rats, matings between two carriers consisted of parents identified or not identified (" unknown") as carriers previously. In the latter case, analysis was made under the assumption that among the "unknowns", the frequency of a non-carrier $(h)$ is 0.33 . The $\chi^{2}$ test for $h=0.33$ is not significant in all cases. For matings between the "unknowns", the analysis was performed on the litters with at least one obese rat.

From matings between two carriers, a total of 397 litters of Zucker rats and a total of 32 litters of Koletsky rats were analysed. The segregation frequency in both cases, the Zucker rats as well as the Koletsky rats, is not significantly different from $P=0.25$ expected from a recessive trait.

A second type of mating of Zucker rats was between a male fatty rat and a female carrier (previously identified or "unknown"). This was achieved by controlling the obesity of the fatty rat through diet restriction. When a male fatty rat grew to 275 to $300 \mathrm{~g}$ of body weight, he was given only $15 \mathrm{~g}$ of food a day. This is about half of the food consumption of a fatty rat when fed ad libitum. With the above manipulation, we were able to make 
Table 1

Segregation analysis*

Type of matings

No. of Total Total

litters affected normal $p \quad \chi_{p}^{2} \quad h \quad \chi_{h}^{2}$

I. Zucker rats $\times$ Zucker rats

1. Carrier $x$ carrier

A. Both parents identified as carriers

B. One parent identified as a carrier, one parent unknown

C. Both parents unknown

259

244

793

$0 \cdot 25$

$1 \cdot 196$

329

1766

$\begin{array}{ll}0.25 & 0.605 \\ 0.25 & 2.512\end{array}$

0.33

0.001

2. Carrier $\times$ fatty

A. Carrier identified

B. Carrier not identified

$15 \quad 42$

42

48

$0.50 \quad 0.400$

$0.50 \quad 1 \cdot 134$

$\overline{0.33}$

$\overline{0 \cdot 404}$

II. Koletsky rats $\times$ Koletsky rats

1. Carrier $\times$ carrier

A. Both parents identified

B. One parent identified as a carrier, one parent unknown

III. Zucker rats $\times$ Koletsky rats
1. Carrier $\times$ carrier

5

11

43

$0.25 \quad 0 \cdot 617$

27

$49 \quad 233$

$0 \cdot 25$

0.758

0.33

0.001

* The results presented in this table are from analysis performed on data pooled from both male and female progeny.

about 10 male fatty rats fertile. Female fatty rats undergoing the same treatment remained infertile. The segregation ratio from this type of mating (carrier $\times$ fatty) is not significantly different from $P=0.50$ expected from a recessive trait.

Mating a Koletsky carrier with a Zucker carrier produced obese $F_{1}$ rats indicating that the gene that controls obesity in the Zucker rats and the
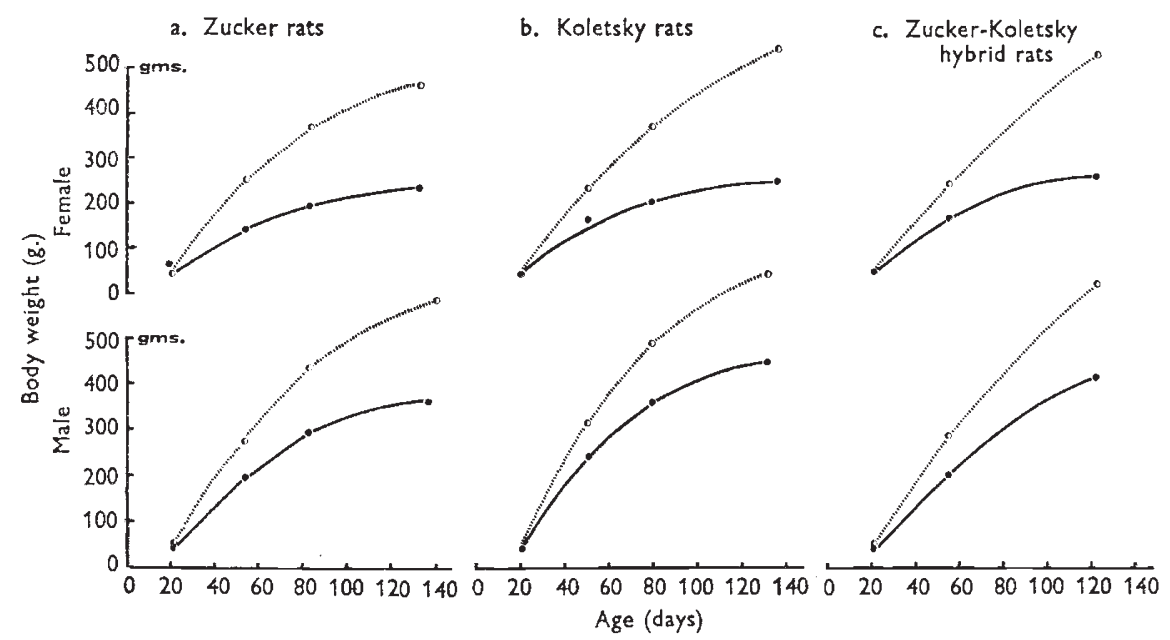

FIG. 1.-Growth curves of female (upper sections) and male (lower sections), normal (solid line) and obese (dashed line) Zucker rats (a), Koletsky rats (b) and Zucker-Koletsky hybrid rats (c). Each curve represents the mean body-weight change of six animals. The standard errors of the mean are generally less than 10 per cent of the mean and are not indicated in the graph.

$38 / 3-G$ 
gene that controls obesity in the Koletsky rats are located at the same locus. The growth curves of male and female, normal and obese Zucker rats, Koletsky rats and Zucker-Koletsky hybrid rats are depicted in fig. I. The Koletsky obese rats and the Zucker-Koletsky hybrid obese rats were heavier, and presumably more obese, than the Zucker obese rats. This difference could be due to a difference between the two strains and/or diets since the male and female normal rats of the Koletsky strain were respectively heavier than the male and female rats of the Zucker strain.

The segregation frequency from four Zucker carrier $\times$ Koletsky carrier matings is not significantly different from 0.25 expected from a recessive trait (table 1). This indicates that the obesity in the Zucker-Koletsky hybrid stock is also inherited in a recessive manner.

In all three types of rats, Zucker, Koletsky, and Zucker-Koletsky, our results showed no difference referable to sex.

\section{Discussion}

Our breeding data on Zucker rats support Zucker and Zucker's original hypothesis that the obesity in these rats is due to a single gene mutation, fatty, and that the trait is inherited in a recessive manner (Zucker and Zucker, 196I). Our data on Koletsky rats also support Koletsky's initial observation that the obesity in the Koletsky rats was controlled by a single gene and it is inherited recessively (Koletsky, 1973).

Our data indicate further that the two genes are related. To interpret our results that a Zucker heterozygote $(f a / F a)$ mated to a Koletsky heterozygote $(f \mid F)$ produced some progeny that became obese and the obesity in the Zucker-Koletsky hybrid rats is also inherited recessively, the simplest hypothesis would be that $f$ and $f a$ are alleles at the same locus.

Our present knowledge does not allow us to conclude whether $f$ and $f a$ are the same allele or two different alleles. The Zucker obese rats and the Koletsky obese rats are phenotypically very different; therefore, $f$ and $f a$ may be two different alleles. However, the Zucker rat and the Koletsky rat were on different diets and have entirely different strain backgrounds which may greatly affect the expression of the same allele (Hummel, Coleman and Lane, 1972; Coleman and Hummel, 1973). The Koletsky rats are hypertensive whereas the Zucker rats are not. The phenotypic expression of the same allele may also be influenced by a second phenotype, hypertension, in the Koletsky rats.

To determine whether $f$ and $f a$ are identical or not, it is imperative to place both alleles on the same strain background. Until this is done and that $f$ and $f a$ are proven to be identical, we propose that $f$ be renamed $f a^{k}$ (for fatty Koletsky) to signify that $f$ and $f a$ are at the same locus.

The authors wish to thank Miss Wilma Young for her excellent technical assistance; Ms Bernice Dunscomb for preparing, and Dr G. L. Wolff, National Center for Toxicological Research, for reviewing this manuscript. The work of P. L. Y. was partially supported by the Indiana University Human Genetics Center, PHS GM-21054 and Indiana University Hypertension Center PHS HL-14159.

\section{REFERENCES}

BRAY, G. A., AND YORK, D. A. 1971. Genetically transmitted obesity in rodents. Physiol. Rev., 51, 598-646. 
COleman, D. L., AND HUMmel, K. P. 1973. The influence of genetic background on the expression of the obese (ob) gene in the mouse. Diabetologia, 9, 287-293.

HUMMEL, K. P., COleman, D. L., AND laNe, P. W. 1972. The influence of genetic background on expression of mutations at the diabetes locus in the mouse. I. C57BL/KsJ and C57BL/6J strains. Biochem. Genet., 7, 1-13.

KOLETSKY, s. 1973. Obese spontaneously hypertensive rats-a model for study of atherosclerosis. Exp. Mol. Pathol., 19, 53-60.

Koletsky, s. 1975. Pathologic findings and laboratory data in a new strain of obese hypertensive rats. Am. 7. Pathol., 80, 129-142.

MORTON, N. E. 1958a. Genetic tests under incomplete ascertainment. Am. J. Hum. Genet., $11,1-16$.

MORTON, N. E. 1958b. Segregation analysis in human genetics. Science, 127, 79-80.

OKAмото, к., AND AOKI, к. 1963. Development of a strain of spontaneously hypertensive rats. Jap. Circulation $7 ., 27,282-293$.

YEN, T. T., SHAW, w. N., AND YU, P. L. 1976. Genetics of obesity in Koletsky rats. Genetics, 83, S85.

zucker, L. M., AND zucker, T. F. 1961. Fatty, a new mutation in the rat. J. Heredity, 52 , 275-278.

ZUCKeR, T. F., AND ZUCKer, L. M. 1963. Fat accretion and growth in the rat. $7 . \mathcal{N} u t r ., 80$, 6-19. 